\title{
Meccanismi immunologici alla base della tolleranza immunitaria
}

\section{Antonio Uccelli}

Centro per la Ricerca e la Cura della Sclerosi Multipla, Dipartimento di Neuroscienze, Riabilitazione, Oftalmologia, Genetica e Scienze Materno-Infantili (DINOGMI) - Università di Genova; IRCCS AOU San Martino - IST - Istituto Nazionale per la Ricerca sul Cancro, Genova; Centro di Eccellenza per la Ricerca Biomedica (CEBR) - Università di Genova

\section{Eziopatogenesi della sclerosi multipla}

La sclerosi multipla (SM) è una malattia infiammatoria demielinizzante del sistema nervoso centrale (SNC) causata da cellule T autoreattive che riconoscono antigeni "self", tra cui antigeni della mielina, e infiltrano il SNC. L'ingresso di cellule T naïve nel SNC è limitata dalle "tight junctions" tra le cellule endoteliali che compongono la barriera ematoencefalica (BEE). Le cellule T attivate esprimono molecole di adesione sulla loro superficie che permettono l'extravasazione attraverso la BEE. Pertanto, il primo evento nella patogenesi della SM è la rottura della tolleranza immunologica che permette l'attivazione, nel sangue periferico, di cellule $\mathrm{T}$ naïve specifiche per antigeni "self". Studi epidemiologici e genetici

\section{Indirizzo per la corrispondenza:}

Antonio Uccelli

e-mail: auccelli@neurologia.unige.it

Accettato: 08/10/2015 - Pubblicato online: 23/11/2015

(C) 2015 The Authors. This article is published by HPS Srl and licensed under Creative Commons Attribution-NC-ND 4.0 International (CC BY-NC-ND 4.0). Any commercial use is not permitted and is subject to Publisher's permissions. Full information is available at www.aboutpharma.com/publishing/riviste/aboutopen/ indicano che la SM è scatenata in soggetti geneticamente predisposti a seguito dell'esposizione a fattori ambientali, solo parzialmente conosciuti, che sono probabilmente responsabili per la perdita della tolleranza immunologica e l'attivazione, in periferia, delle cellule $\mathrm{T}$ autoreattive. Una volta che le cellule $\mathrm{T}$ autoreattive sono entrate nel SNC, esse incontrano le cellule presentanti l'antigene (antigen presenting cell, APC), quali cellule dendritiche (dendritic cell, DC) e macrofagi, soprattutto a livello dei plessi corioidei e degli spazi subaracnoidei, e macrofagi infiltranti e microglia nel parenchima, che sono in grado di presentare epitopi di proteine della mielina sintetizzate all'interno del SNC. La riattivazione di queste cellule all'interno del SNC induce una serie di meccanismi effettori, quali il rilascio di citochine e chemochine che amplifica la risposta infiammatoria attraverso il reclutamento di altre cellule infiammatorie (inclusi linfociti $\mathrm{T}$, linfociti $\mathrm{B}$ e macrofagi) che, alla fine, portano al danno della mielina, alla degenerazione degli assoni e infine alla distruzione tissutale [1] (Figura 1). Gran parte delle nostre conoscenze della patogenesi della SM si basano su studi che utilizzano il modello animale dell'encefalomielite autoimmune sperimentale (experimental au- 


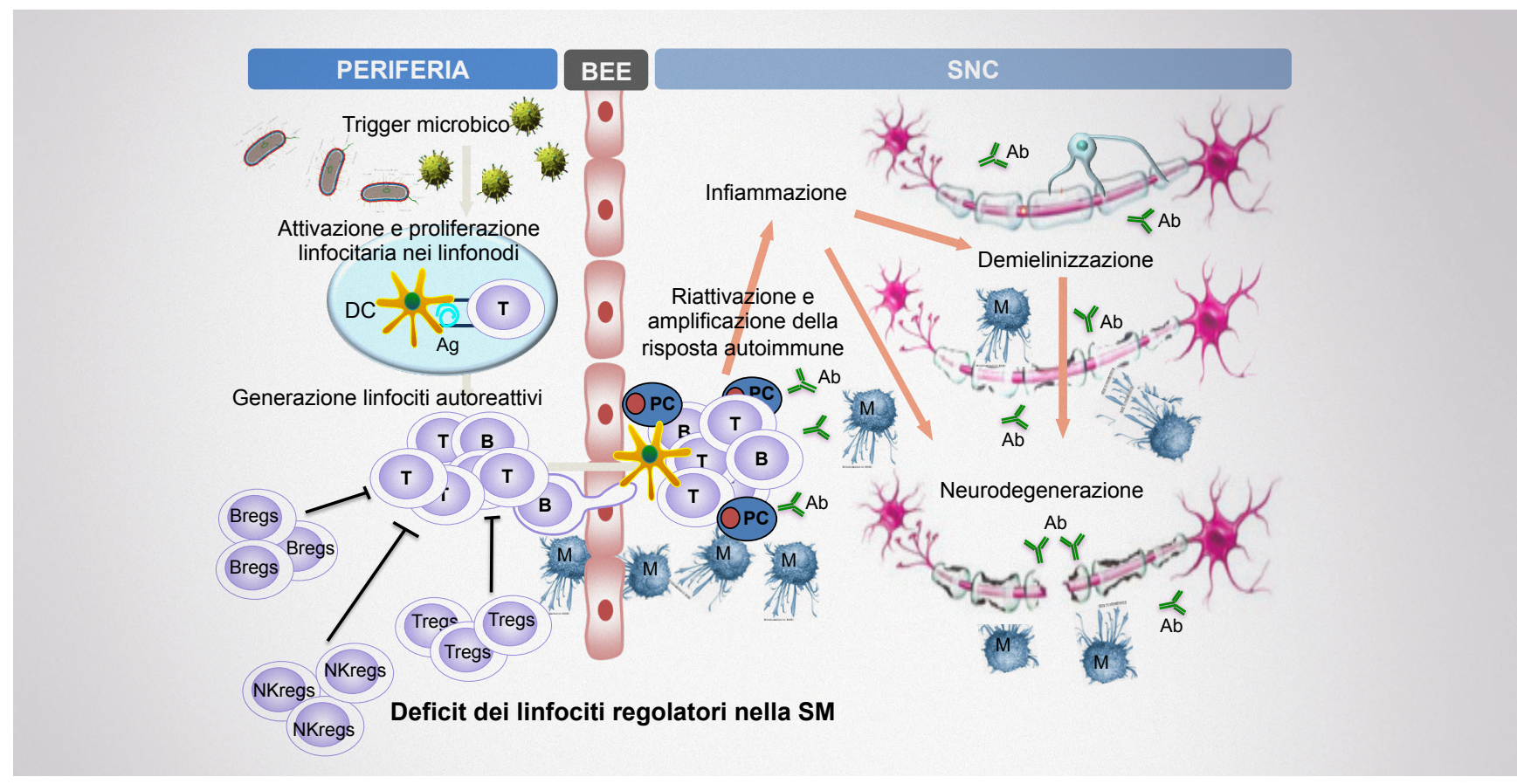

Figura 1. Rottura della tolleranza immunologica nella sclerosi multipla (SM). Ab, anticorpo; Ag, antigene; B, linfocita B; BEE, barriera ematoencefalica; Breg, linfociti B regolatori; DC, cellula dendritica; M, macrofago; PC, plasmacellula; SNC, sistema nervoso centrale; T, linfocita T; Treg, linfociti T regolatori.

toimmune encephalomyelitis, EAE). L'EAE è indotta negli animali di laboratorio (in genere nei roditori) attraverso l'immunizzazione con antigeni mielinici in grado di stimolare una risposta immunitaria diretta contro proteine del SNC [2]. L'EAE può anche essere indotta passivamente mediante il trasferimento di cellule $\mathrm{T} \mathrm{CD}^{+}$specifiche per la mielina - ottenute da un animale immunizzato - in un animale sano [3]. La capacità di indurre EAE attraverso il trasferimento passivo di cellule $\mathrm{T}$ specifiche per la mielina rafforza l'idea che la SM sia una malattia autoimmune mediata dai linfociti T CD4+. Tuttavia, l'analisi del tessuto ottenuto da pazienti affetti da SM ha dimostrato che le cellule $\mathrm{T} \mathrm{CD}^{+}$sono più abbondanti nelle lesioni rispetto alle cellule $\mathrm{T} \mathrm{CD}^{+}$. Inoltre, è stato osservato che, nel sangue e nel liquido cefalorachidiano di soggetti con SM, è possibile osservare l'espansione clonale di cellule $\mathrm{T} \mathrm{CD}^{+}$più frequentemente rispetto a quella delle cellule T $\mathrm{CD}^{+}$[4], suggerendo che anche i linfociti $\mathrm{T} \mathrm{CD}^{+}$giochino un ruolo chiave nella patogenesi della SM.

\section{Meccanismi della tolleranza immunologica centrale e periferica}

La prima occasione per eliminare cellule T potenzialmente autoreattive avviene nel timo, dove i timociti, i precursori dei linfociti $\mathrm{T}$, che interagiscono ad alta avidità con antigeni "self" presentati dalle APC, vengono eliminati attraverso un meccanismo detto di "delezione clonale". I timociti, le cui interazioni con le APC non sono abbastanza forti per indurre morte cellulare, ma sono sufficienti per indicare un livello inaccettabile di autoreattività, sono inattivati funzionalmente attraverso meccanismi di "anergia", revisione del T-cell receptor (TCR) o acquisiscono caratteristiche funzionali di "linfociti $\mathrm{T}$ regolatori Foxp $3^{+}$". È importante considerare inoltre che la maggior parte delle proteine presenti nella mielina centrale non si trovano nella mielina periferica, e pertanto sono considerate antigeni tessuto-specifici, risultando nascosti al sistema immunitario ("antigeni criptici"). Alcune cellule epiteliali del timo possono esprimere 
antigeni tessuto-specifici permettendo di indurre tolleranza centrale verso auto-antigeni ed eliminando il repertorio $\mathrm{T}$ cellulare della maggior parte delle cellule autoreattive. Nonostante i numerosi meccanismi di tolleranza centrale, molte cellule $\mathrm{T}$ autoreattive sfuggono a questi processi regolatori e sono presenti nel sangue periferico. Per esempio, cellule T autoreattive possono indurre autoimmunità nel caso la frequenza di complessi auto-antigene/MHC (Major Histocompatibility Complex) sia più alta in periferia rispetto al timo oppure una modificazione post-traslazionale di un auto-antigene avvenga in periferia tanto da aumentare l'affinità del TCR di un linfocita per il complesso MHC/auto-antigene. Inoltre, antigeni che sono espressi in fasi più tardive dello sviluppo non possono essere presentati dall'epitelio timico quando i timociti sono sottoposti a selezione negativa; essi sfuggono pertanto ai meccanismi di tolleranza centrale. In questo modo, gli antigeni espressi più tardivamente nel nostro organismo potrebbero diventare il bersaglio di cellule $\mathrm{T}$ autoreattive maturate nel timo ed entrate in circolazione prima dell'espressione dell'auto-antigene. Infine, una certa tolleranza verso antigeni "self" potrebbe essere necessaria se si considera che potrebbe non essere possibile esercitare una rigorosa tolleranza centrale in grado di eliminare tutte le cellule $\mathrm{T}$ autoreattive senza rischiare di generare un repertorio $\mathrm{T}$ cellulare periferico che sia incapace di esibire la più ampia specificità verso i patogeni.

Poiché la tolleranza centrale non è completamente efficiente nel prevenire l'autoimmunità, esistono meccanismi di controllo che si esercitano in periferia, la cosiddetta "tolleranza immunologica periferica". Tra questi meccanismi, che sono generalmente indotti quando una cellula $\mathrm{T}$ riconosce il complesso MHC/auto-antigene espresso sulla superficie di una cellula dendritica non attivata, va ricordata la delezione clonale delle cellule $\mathrm{T}$, l'induzione di anergia linfocitaria e la sotto-regolazione del TCR e/o delle molecole co-stimolatorie (per esempio CD80, CD86 ecc.) [5]. Infine, sempre maggiore importanza nel mantenimento della tolleranza periferica è data a numerosi tipi di cellule con funzioni regolatorie.

\section{Linfociti regolatori}

Linfociti T regolatori (Treg) sono essenziali per mantenere l'omeostasi del sistema immunitario, limitando l'entità delle risposte effettrici e permettendo la creazione della tolleranza immunologica. Sono stati identificati due tipi principali di cellule $\mathrm{T}$ regolatorie: quelle naturali, che si sviluppano nel timo, e quelle inducibili. Le $\mathrm{T}$ regolatorie inducibili (iTreg) sono linfociti $\mathrm{CD}_{4}{ }^{+}$che esprimono il fattore di trascrizione Foxp3 e si sviluppano al di fuori del timo in diverse condizioni [6]. Più recentemente è stato dimostrato che linfociti B regolatori (Breg) contribuiscono al mantenimento della tolleranza periferica limitando le risposte infiammatorie eccessive che si verificano durante le malattie autoimmuni o che possono essere causate da infezioni non controllate [7]. Più recentemente è stata dimostrata un'azione immunoregolatoria anche da parte di una popolazione di cellule natural killer (NK) esprimente alti livelli del recettore $\mathrm{CD} 56$ (NK CD56 $6^{\text {bright }}$ ) [8]. Studi recenti hanno dimostrato che, benché la frequenza delle cellule regolatorie nei pazienti con SM sia simile a quella dei controlli sani, queste possono essere funzionalmente incapaci di controllare cellule autoreattive in soggetti con SM [9].

\section{Rottura della tolleranza immunologica nella sclerosi multipla}

I risultati ottenuti nel modello sperimentale dell'EAE hanno supportato il concetto che le cellule $\mathrm{T}$ che producono interferone- $\gamma$ (IFN $\gamma$ ) e tumor necrosis factor- $\alpha$ (TNF $\alpha)$, cioè i linfociti Th1, siano considerate come i principali effettori dell'autoaggressione al SNC. Tuttavia, dati più recenti fanno pensare che $i$ linfociti Th17, che producono interleuchina (IL)-17, siano coinvolti nella patogenesi della malattia [10]. Dati ottenuti nell'EAE suggeriscono che le cellule immunitarie coinvolte nell'immunosorveglianza del SNC entrino attraversando il plesso corioideo grazie al recettore CCR6 espresso sulle cellule Th17 e il suo ligando, la chemochina CCL20, espressa sull'epitelio 
del plesso corioideo [11]. Se questo modello fosse applicabile anche alla SM, potremmo ipotizzare che, nel contesto di un difetto dell'immunoregolazione periferica, cellule Th17 autoreattive siano attivate nel sangue periferico e possano migrare, attraverso il plesso corioideo, nel SNC. Una volta all'interno dei ventricoli, le cellule autoreattive potrebbero penetrare nel parenchima periventricolare o proseguire la circolazione negli spazi subaracnoidei fino a quando accedono alla pia e alla corteccia subpiale in cerca dell'antigene target (Figura 2). Le citochine IL-17 e IL-22 rilasciate dalle cellule Th17 aumentano la permeabilità della BEE e danno inizio a una cascata di eventi infiammatori che permettono la penetrazione delle cellule immunitarie, tra cui altri linfociti Th17 e Th1 autoreattivi, linfociti $\mathrm{T} \mathrm{CD}^{+}$citotossici, cellule $\mathrm{B}$, plasmacellule e macrofagi, che contribuiscono a formare gli infiltrati perivascolari e causano la demielinizzazione e, nel tempo, la degenerazione assonale.

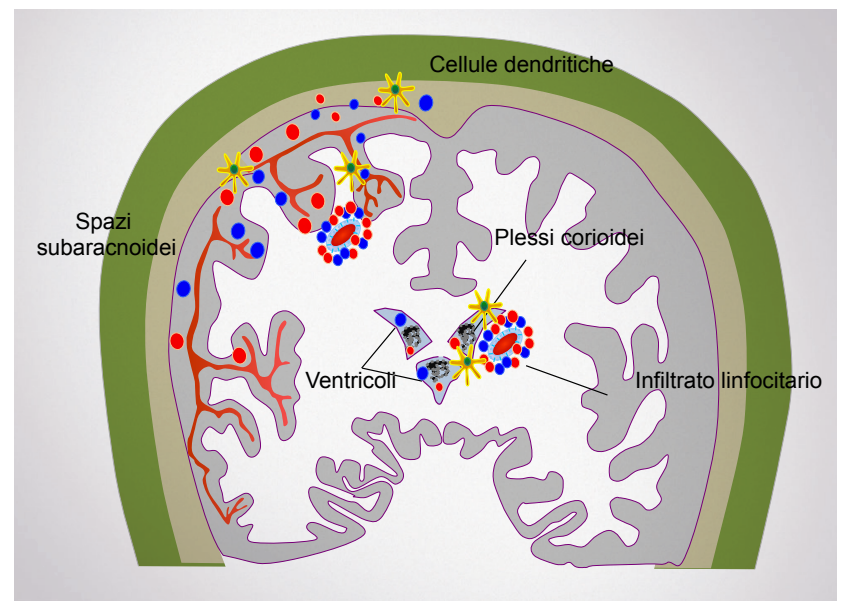

Figura 2. Migrazione dei linfociti T attraverso i plessi corioidei e circolazione negli spazi subaracnoidei.

\section{Quali sono gli auto-antigeni?}

Fino a oggi è stato difficile identificare auto-antigeni (mielinici e no) che siano riconosciuti dalle cellule autoreattive nelle persone con SM, diversamente da quanto avviene in altre malattie neurologiche autoimmuni, quali la miastenia gravis o la neuromielite ottica, dove sono stati identificati anticorpi ad alta affinità che riconoscono gli antigeni "self" (rispettivamente il recettore per l'acetilcolina e la proteina acquaporina 4). Tuttavia, il consenso generale è che non siano uno solo, bensì diversi, gli antigeni patogenetici coinvolti in questa malattia. Inoltre, anche se le cellule autoreattive potrebbero essere inizialmente specifiche per un antigene particolare, è probabile che nel corso dello sviluppo della risposta autoimmune si verifichi una diversificazione della risposta verso target differenti all'interno della stessa proteina o anche verso proteine diverse (fenomeno dell'epitope spreading) [12]. È significativo che cellule T autoreattive siano state descritte sia nei soggetti sani che nei pazienti con SM. L'attivazione nel sangue periferico di queste cellule autoreattive da parte di fattori ambientali ancora solo parzialmente conosciuti, ma potenzialmente di origine microbica oltre che di altro tipo, insieme a un deficit delle cellule regolatorie che le controllano, ne permette la migrazione nel SNC, dove possono essere attivate da cellule presentanti l'antigene nel tessuto (microglia, macrofagi infiltranti e cellule dendritiche nei plessi corioidei e negli spazi subaracnoidei locali) e possono contribuire all'infiammazione e al danno tissutale.

\section{Conclusioni}

La sempre maggiore conoscenza dei meccanismi patogenetici della SM, così come dei meccanismi che controllano la tolleranza immunologica, è stata di fondamentale importanza per lo sviluppo di trattamenti per la SM. Molti dei farmaci attualmente in uso sono in grado di interferire con questi meccanismi e restaurare, almeno in parte, la tolleranza immunologica, per esempio attraverso l'espansione di cellule a funzione regolatoria. Lo sviluppo di tecniche sempre più sofisticate per studiare l'impatto di questi farmaci sulle cellule del sistema immunitario sarà di fondamentale importanza per verificarne il beneficio clinico e controllarne i possibili rischi. 


\section{Disclosures}

L'autore dichiara di avere ricevuto un contributo finanziario per la sua attività di consulente o relatore da parte di Biogen, Novartis, Merck Serono Teva, Sanofi-Aventis, Genzyme e Roche e di avere avuto un supporto finanziario per le proprie ricerche da parte di Biogen, Merck Serono e Novartis.

\section{Bibliografia}

1. Goverman J. Autoimmune T cell responses in the central nervous system. Nat Rev Immunol. 2009;9(6):393-407.

2. Stromnes IM, Goverman JM. Active induction of experimental allergic encephalomyelitis. Nat Protoc. 2006;1(4):1810-9.

3. Stromnes IM, Goverman JM. Passive induction of experimental allergic encephalomyelitis. Nat Protoc. 2006;1(4):1952-60.

4. Friese MA, Fugger L. Autoreactive CD8+ T cells in multiple sclerosis: a new target for therapy? Brain. 2005;128(Pt 8):1747-63.

5. Steinman RM, Hawiger D, Nussenzweig MC. Tolerogenic dendritic cells. Annu Rev Immunol. 2003;21:685-711.
6. Bilate AM, Lafaille JJ. Induced CD4+Foxp3+ regulatory T cells in immune tolerance. Annu Rev Immunol. 2012;30:733-58.

7. Mauri C, Bosma A. Immune regulatory function of B cells. Annu Rev Immunol. 2012;30:221-41

8. Jiang W, Chai NR, Maric D, Bielekova B. Unexpected role for granzyme $\mathrm{K}$ in CD56bright NK cell-mediated immunoregulation of multiple sclerosis. J Immunol. 2011;187:781-90.

9. Nylander A, Hafler DA. Multiple sclerosis. J Clin Invest. 2012;122(4):1180-8.

10. Korn T, Bettelli E, Oukka M, Kuchroo VK. IL-17 and Th17 Cells. Annu Rev Immunol. 2009;27:485-517.

11. Reboldi A, Coisne C, Baumjohann D, et al. C-C chemokine receptor 6-regulated entry of TH-17 cells into the CNS through the choroid plexus is required for the initiation of EAE. Nat Immunol. 2009; 10(5):514-23

12. McMahon EJ, Bailey SL, Castenada CV, et al. Epitope spreading initiates in the CNS in two mouse models of multiple sclerosis. Nat Med. 2005;11(3):335-9. 